\title{
Lifetime and Coverage Guarantees Through Distributed Coordinate-Free Sensor Activation
}

\author{
Gaurav S. Kasbekar \\ Department of Electrical and \\ Systems Engineering \\ University of Pennsylvania \\ Philadelphia, PA, USA \\ kgaurav@seas.upenn.edu
}

\author{
Yigal Bejerano \\ Bell-Labs, Alcatel-Lucent \\ Murray Hill, NJ, USA \\ bej@research.bell- \\ labs.com
}

\author{
Saswati Sarkar \\ Department of Electrical and \\ Systems Engineering \\ University of Pennsylvania \\ Philadelphia, PA, USA \\ swati@seas.upenn.edu
}

\begin{abstract}
Wireless Sensor Networks are emerging as a key sensing technology, with diverse military and civilian applications. In these networks, a large number of sensors perform distributed sensing of a target field. Each sensor is a small battery-operated device that can sense events of interest in its sensing range and can communicate with neighboring sensors. A sensor cover is a subset of the set of all sensors such that every point in the target field is in the interior of the sensing ranges of at least $k$ different sensors in the subset, where $k$ is a given positive integer. The lifetime of the network is the time from the point the network starts operation until the set of all sensors with non-zero remaining energy does not constitute a sensor cover. An important goal in sensor networks is to design a schedule, that is, a sequence of sensor covers to activate in every time slot, so as to maximize the lifetime of the network. In this paper, we design a polynomial-time, distributed algorithm for maximizing the lifetime of the network and prove that its lifetime is at most a factor $O(\log n * \log n B)$ lower than the maximum possible lifetime, where $n$ is the number of sensors and $B$ is an upper bound on the initial energy of each sensor. Our algorithm does not require knowledge of the locations of nodes or directional information, which is difficult to obtain in sensor networks. Each sensor only needs to know the distances between adjacent nodes in its transmission range and their sensing radii. In every slot, the algorithm first assigns a weight to each node that is exponential in the fraction of its initial energy that has been used up so far. Then, in a distributed manner, it finds a $O(\log n)$ approximate minimum weight sensor cover which it activates in the slot. Our simulations reveal that our algorithm substantially outperforms several existing lifetime maximization algorithms.
\end{abstract}

\section{Categories and Subject Descriptors}

C.2.1 [Network Architecture and Design]: Wireless communication

Permission to make digital or hard copies of all or part of this work for personal or classroom use is granted without fee provided that copies are not made or distributed for profit or commercial advantage and that copies bear this notice and the full citation on the first page. To copy otherwise, to republish, to post on servers or to redistribute to lists, requires prior specific permission and/or a fee.

MobiCom'09, September 20-25, 2009, Beijing, China.

Copyright 2009 ACM 978-1-60558-702-8/09/09 ...\$10.00.

\section{General Terms}

Algorithms, Design, Performance, Theory

\section{Keywords}

Wireless Sensor Networks, Network Lifetime, Coverage, Approximation Algorithms, Distributed Algorithms, CoordinateFree

\section{INTRODUCTION}

Recent advances in wireless communications and electronics have enabled the development of low-cost sensor nodes [12]. Each sensor node is capable of sensing specific events in its vicinity and of communicating with adjacent nodes. Thus, for event sensing applications, a large number of sensor nodes are deployed in a distribution area and they collaborate to form an ad-hoc network, referred to as a wireless sensor network (WSN). WSNs have the potential to become the dominant sensing technology in many civilian and military applications, such as intrusion detection, environmental monitoring, object tracking, traffic control, and inventory management. In many of these applications, WSNs need to monitor the target field for detecting events of interest, e.g., entrance of an intruder in an intrusion detection application.

Wide-spread deployment of WSNs in target field monitoring is being deterred by the energy consumed in the monitoring process. The challenge is compounded by the fact that the sensors are battery-powered and owing to size limitations the sensors can only be deployed with low-lifetime batteries, most of which are not rechargeable. Thus, a sensor ceases to function (e.g., monitor) once its battery expires, and oftentimes, sensors whose batteries have expired can not be easily replaced owing to logistics issues such as remoteness or inaccessibility of distribution areas. Thus, the success of the WSN technology is contingent upon developing strategies for intelligently using the available sensors so as to maximize the duration for which the entire target field is monitored by sensors. This duration, referred to as the network lifetime, is an important performance metric for the network as the coverage of the entire target field is essential for reliable detection of events of interest.

Owing to large scale availability of low cost sensors, sensors are often deployed with some redundancy, that is, several locations in the target field can be monitored by multiple sensors. Lifetime of the WSNs can be substantially enhanced by intelligently activating the sensors that monitor the target field at any given time. We seek to maxi- 
mize the lifetime of sensor networks by designing algorithms that dynamically activate sensors based on their residual energy content. The algorithm we develop is completely distributed, does not need to know the coordinates of any sensor, and provides provable guarantees on the attained lifetimes.

\subsection{Related Literature}

Coverage, connectivity and lifetime maximization for WSNs have received considerable attention in the last few years. Comprehensive surveys can be found in [14, 15]. Most of the existing papers focus on the coverage and connectivity aspects $[2,16,17,18,6,10,19,20,21]$, and typically propose computational geometry based approaches for discovering coverage holes and ensuring connectivity. An interesting connectivity property has been proved in [20, 21] that shows that if the trasmission radius of each node is at least twice of its sensing radius, then coverage implies connectivity of the sensor network. We make the same assumption, and therefore seek to maximize lifetime while guaranteeing coverage without explicitly considering connectivity.

We now summarize the papers that propose topology control solutions that maximize the network lifetime by scheduling the active periods of the sensors, while preserving coverage and connectivity requirements. In [13] Cardei et al. address the problem of lifetime maximization when only a given set of targets needs to be covered. They showed that the problem is NP-hard and provided heuristic sensor activation algorithms based on linear programming relaxations. They also proposed a greedy heuristic activation scheme that at each round seeks the minimal set of sensors that covers all the targets. They evaluated the lifetimes attained by the heuristic solutions using simulations, but did not provide provable guarantees on the lifetimes of these schemes. To our knowledge, the only scheme that provides guarantees on the network lifetime is the one proposed by Berman et al. [11]. They have provided a centralized algorithm that attains a network lifetime which is within $O(\log n)$ of the maximum possible lifetime, where $n$ is the number of sensors. This algorithm determines how to activate sensors based on an approximate solution of a linear program that requires complete knowledge of network topology, coordinates of sensor locations and initial energy of sensors. Such linear programs can clearly be solved only by a central entity that knows all of the above, which is hard to realize in practice. Also, the sensors rarely know their precise locations since WSNs usually do not have access to global positioning systems (GPS). Several sensor positioning systems $[23,24]$ have been proposed in the literature for learning the locations, without manual configuration or the use of GPS receivers. However, they provide only coarse location estimations in practical settings [25]. Note that several coverage verification algorithms that do not assume knowledge regarding the locations of the sensors exist $[17,16,10,3]$, but these papers do not provide any guarantee on the network lifetime. Our contribution is to provide a distributed, coordinate-free sensor activation scheme that provides provable guarantees on the network lifetime.

Finally, Wu et al. [22] considered a different notion of lifetime in a recent paper: the maximum time until which all nodes in the data aggregation tree of choice remain operational, (a node in this case consumes energy only during communication). Since we focus on the energy consumed in sensing, our notion of lifetime, the problem formulation and solution techniques differ substantially.

\subsection{Our Contribution}

The contribution of this paper is two-fold.

First, we present the first coordinate-free distributed scheme that provides provable approximation guarantees on network lifetime, while providing strict coverage guarantees. This is a surprising result since the sensors are not aware of their coordinates in a global coordinate system, and are therefore oblivious to their locations relative to each other and to the target field. To overcome this challenge we assume that the sensor distribution area is slightly larger than the area that needs to be monitored. The sensors are divided into periphery nodes that are located near the boundary of the distribution area and internal nodes that are internal to this area. The target field that our scheme is committed to monitor is taken as the closure of the area covered by the internal nodes. Our scheme at each time slot selects a subset of sensors for monitoring the target field that ensure $k$-coverage of the entire target field, for a given integer $k \geq 1$, and different subsets may be selected in different slots. The selection process relies on two key steps: (i) each sensor is assigned a weight that is an exponentially increasing function of the energy it has consumed so far (ii) the set of sensors that has the minimum total weight, or an approximation thereof, among all those that cover the entire target field is activated. This selection process balances the monitoring load on all the sensors, and preferentially selects in each slot the sensors with high residual energy. We demonstrate that the algorithm can be executed using distributed computations that do not need to know the locations of the sensors.

Second, we prove that the lifetime of the network when this algorithm is used is at least $1 / O((\log n)(\log n B))$ of the optimal solution, where $n$ is the number of sensors and $B$ is a bound on the initial energy level of the nodes. We prove this approximation ratio, by extending to this problem the exponential-function technique, originally developed by Aspnes et al. [26] in the context of online machine scheduling and virtual circuit routing and later used by Awerbuch et al. [4] in on-line virtual circuit routing. Thus, our algorithm attains a provable guarantee which is only slightly worse as compared with the best available centralized performance guarantee till date, presented in [11]. We demonstrate via simulations that our scheme attains a significantly higher lifetime than several other existing schemes $[11,21,13]$.

\section{PRELIMINARY}

\subsection{Network Model}

We consider a wireless sensor network (WSN) consisting of a set $S$ of $n$ sensors that are also called nodes. Each node $u \in S$ can sense events of interest in its sensing range and communicate with nodes in its transmission range. We make the natural assumption that there are no two sensors at the same location. Also, each sensor $u \in S$ has a unique identification number, denoted by $I D(u)$. The sensors are distributed over a large 2-dimensional area. We refer to the region obtained by the union of the sensing ranges of all the sensors as the distribution area and it subsumes the region that needs to be monitored by the sensors, referred to as the monitoring area. The latter is typically significantly larger than the sensing range of a single sensor. 
We assume that the sensing and transmission ranges of a node $u$ are open discs, centered at $u$, with radii $r_{u}$ and $R_{u}$ respectively, where $R_{u}>r_{u}$. Let $\hat{r}=\max _{u \in S} r_{u}$, and $\hat{R}=$ $\min _{u \in S} R_{u}$. The boundary of the sensing range of any node $u$ is a circle, which we refer to as the sensing border of node $u$. Let $d_{u, v}$ denote the Euclidean distance between nodes $u$ and $v$. Nodes $u$ and $v$ are termed adjacent or neighbors if they are included in the transmission range of each other. Let $N_{u}$ be the set of neighbors of $u$.

We assume that nodes only have localized distance information. Specifically, each node $u$ knows (a) $r_{u}$, (b) $d_{u, v}$ and $r_{v}$ for each $v \in N_{u}$ and (c) $d_{v, w}$ for each pair $w, v \in N_{u}$ such that $w$ and $v$ are neighbors of each other. Thus, we assume that each node can estimate its sensing radius, and its distances from its neighbors without learning their orientations, and communicates this information to its neighbors. Note that recent studies $[8,9]$ have introduced accurate distance estimation techniques that are applicable to wireless sensors.

We assume that there is a periphery band of width at least $\hat{r}$ between the boundary of the distribution area and the edge of the monitoring area. We distinguish between periphery nodes that are located in the periphery band and internal nodes that lie in the monitoring area. Although the sensors are not aware of their locations, we assume that every sensor knows if it is a periphery or an internal node, for instance by using the mechanisms in $[6,7]$.

The time is divided into time slots and we assume that the sensors have synchronized clocks which notify them at the beginning of each time slot. Sensor $u \in S$ has an initial energy $B_{u}$ and, as a normalization, we assume that each sensor consumes 1 unit of energy in each time slot in which it is active. For saving energy, a sensor may be in a sleep mode, in which it does not communicate with its neighbors nor sense its vicinity. A sensor in sleep mode consumes only negligible amount of energy, which we assume to be zero.

\subsection{The Target Field}

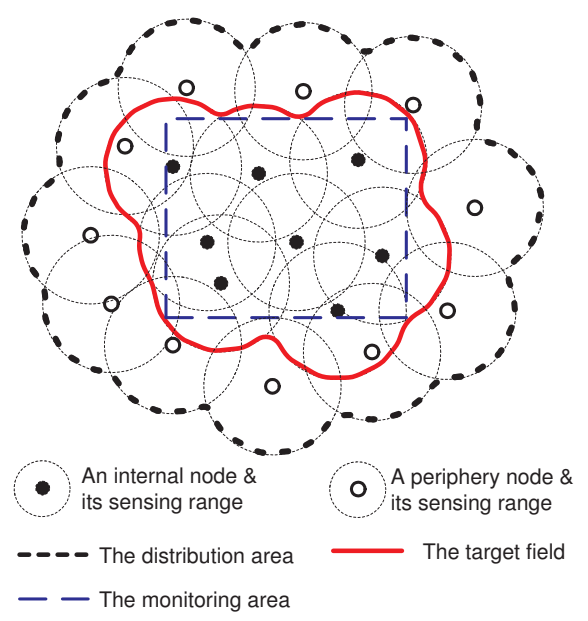

Figure 1: An example of a small WSN and its target field.

Generally speaking, the target field is the area monitored by the system. This area is obviously subsumed in the distribution area and it should contain the monitoring area. Since the sensors are not aware of their locations, they are oblivious to their locations relative to each other and to the monitoring area. Addressing this difficulty, we next provide a precise definition of the target field that our scheme is committed to monitor.

Definition 1 (The TARget Field). The target field is the area defined by the closure ${ }^{1}$ of the union of the sensing ranges of all the internal sensors.

We assume that the target field subsumes the monitoring area. Figure 1 illustrates a small WSN as well as its distribution area, monitoring area and target field.

Given a set $C \subseteq S$ of sensors and a positive integer $k$, we say that a point in the target field is $k$-covered by $C$ if it is in the interior of the sensing range of at least $k$ nodes in $C$. The target field is considered as $k$-covered by $C$ if every point in the target field is $k$-covered by $C$.

Definition 2 (Sensor Cover). A set $C$ of sensors that $k$-covers the target field is termed a sensor cover.

If there does not exist a sensor cover $C$ such that all the nodes in $C$ have non-zero energy, then the network is said to have a coverage hole.

Since the sensing ranges are open discs, no sensor covers its sensing border. Thus, any sensor cover must contain periphery sensors that cover the target-field boundary (see Fig. 1). Thus, sensor activation schemes must consider both internal and periphery nodes.

\subsection{Problem Statement}

We proceed to define the maximum network lifetime problem.

Definition 3 (The Network Lifetime). The network lifetime is the time interval from the activation of the network until the first time at which a coverage hole appears.

Definition 4. (The Maximum Network Lifetime Problem) An activation schedule is a sequence of sensor covers that are activated in successive slots, such that in every slot, each sensor in the activated sensor cover has non-zero energy. The maximum network lifetime problem seeks to find an activation schedule that maximizes the network lifetime.

In [13], the authors prove that the closely-related target coverage version of the maximum network lifetime problem is NP-hard. Moreover, in [10] it has been shown that for a given subset $C \subseteq S$, no coordinate-free algorithm can provably verify whether or not $C$ covers the target field, if $\hat{R}<2 \hat{r}$. So henceforth, we assume that $\hat{R} \geq 2 \hat{r}$ and we present a distributed coordinate-free algorithm for the maximum network lifetime problem with guarantee on the lifetime attained by the calculated schedule.

\subsection{The Intersection Point Concept}

We now present an observation that constitutes a cornerstone in our solution. Consider two sensors $v, z \in S$. The sensors are termed intersecting if their sensing borders intersect (but not tangent to each other). In such case, we say that $v$ intersects with $z$.

\footnotetext{
${ }^{1}$ Recall that the closure of a set $A$ is the smallest closed set that contains $A[27]$.
} 
Property 1 (Intersection). The sensors $v, z \in S$ are intersecting if and only if $d_{v, z}<r_{v}+r_{z}, d_{v, z}+r_{z}>r_{v}$ and $d_{v, z}+r_{v}>r_{z}$.

Note that the sensing borders of any pair $v, z \in S$ of intersecting sensors have exactly two intersection points denoted by $I P(v, z, 1)$ and $I P(v, z, 2)$. Moreover, by Property 1 , since the distance $d_{v, z}<r_{v}+r_{z} \leq 2 \cdot \hat{r}$ and we assume that $\hat{R} \geq 2 \cdot \hat{r}$, any two intersecting sensors $v, z$ are adjacent.

We next show that for calculating a sensor cover we just need to consider sensors that have intersection points on their sensing borders.

Property 2. Consider a sensor cover $C \subset S$ and let $u \in C$ be a sensor without any intersection point on its sensing border. Then the set $C-\{u\}$ is also a sensor cover.

Proof. A necessary condition for the target field to be $k$-covered by $C$ is that every point in the target field is in the interior of the sensing ranges of at least $k$ sensors in $C$. Since we assume that the target field is larger than the sensing range of any single sensor, the set $C$ contains additional sensors beside $u$. Since $u$ 's sensing border does not intersect with that of any other sensor in $C$, then to ensure coverage of the target field, either (a) the sensing range of $u$ does not cover any part of the target field or (b) $u$ 's sensing range is subsumed in the sensing ranges of $k$ other sensors, say $v_{1}, \ldots, v_{k}$, in $C$, and hence $v_{1}, \ldots, v_{k}$ cover the part of the target field covered by $u$. Thus, in both cases $C-\{u\}$ is also a sensor cover.

The next corollary directly follows from Property 2 .

COROllary 1. Let $u \in S$ be a sensor without any intersection point on its sensing border and consider a schedule $\left\{C_{1}, C_{2}, \cdots, C_{L}\right\}$ of sensor covers with network lifetime of $L$ in which node $u$ is active in some slots. Then, the schedule $\left\{\hat{C}_{1}, \hat{C}_{2}, \cdots, \hat{C}_{L}\right\}$, where $\hat{C}_{j}=C_{j}-\{u\}$, also defines a sequence of sensor covers with network lifetime of $L$.

From Corollary 1 it follows that the network lifetime is not affected by ignoring sensors without intersection points on their sensing borders. So henceforth, we will ignore such sensors.

Let $P$ be the set of intersection points that are in the target field, referred to as the $I P$ set. Recall that $P$ contains every intersection point $I P(v, z, i), i=\{1,2\}$, such that at least one of the nodes $v, z \in S$ is an internal node or $I P(v, z, i)$ is in the sensing range of an internal node.

TheOrem 1. Consider a set $C \subset S$ of sensors. The set $C$ is a sensor cover if and only if it $k$-covers every point in the IP set $P$.

Proof. If $C k$-covers the target field, then by definition it $k$-covers every point in $P$.

To prove the converse, suppose $C k$-covers every point in $P$. First, we prove that every point in the target field that lies on the sensing border of some sensor is $k$-covered by $C$. Let $f$ be a point in the target field on sensor $v$ 's sensing border. If $f$ is an intersection point, it is $k$-covered by $C$, by assumption. If not, trace a path from $f$ along $v$ 's sensing border to first reach an intersection point, say $e$. Recall that every sensor that we consider has an intersection point on its sensing border. So there exists such a point $e$. By definition of the target field, $e$ lies in the target field and hence, by assumption, is $k$-covered by $C$. Also, the path traced from $f$ to $e$ did not cross the sensing border of any sensor because the path first reached any intersection point at $e$. So it follows that $f$ is in the interior of the sensing ranges of exactly the same subset of sensors of $C$ as $e$ is in ${ }^{2}$, and hence is $k$-covered by $C$.

Now, let $h$ be any point in the target field. If $h$ lies on the sensing border of some sensor, it is $k$-covered by $C$, as shown above. If not, trace a path from $h$ to first reach the sensing border of some sensor at some point, say $g$. By definition of the target field, $g$ lies in the target field. Hence, as shown above, $g$ is $k$-covered by $C$ and by arguments similar to those in the previous paragraph, $h$ is also $k$-covered by $C$.

Owing to Theorem 1, we henceforth consider as sensor cover any set of sensors that $k$-covers all the intersection points in $P$.

\section{ALGORITHM OVERVIEW}

We now describe the Distributed Lifetime Maximization (DLM) algorithm that we propose. In this section, we present a brief overview of the individual building blocks in DLM, and provide the details in Sections 4, 5 .

Our algorithm consists of an initialization phase and an activation phase. The initialization phase is executed once, at the beginning of the network operation, and informs the nodes of some network-parameters. Every node executes the activation phase at the beginning of each subsequent time slot, and decides whether to activate itself in the slot based only on the state information in its neighborhood. We now describe the above phases, and introduce some new terminologies towards that end.

Consider a sensor cover $C$, and let sensor $u$ have weight $w_{u}$, a positive real number. The weight of the sensor cover $C$ is the sum of the weights of the sensors in $C$, i.e., $\sum_{u \in C} w_{u}$.

Definition 5 (A minimum Weight Sensor COVer). $A$ minimum weight sensor cover is a sensor cover that has the minimum weight among all sensor covers. An $\alpha$-approximate minimum weight sensor cover is one whose weight is at most $\alpha$ times that of the minimum weight sensor cover.

Let $P_{u}$ be the set of intersection points covered by sensor $u$, and $T_{u}$ be the set of sensors $v$ such that sensors $u$ and $v$ cover a common intersection point.

\subsection{Initialization phase}

An initialization phase is executed at the beginning of the network operation, i.e., at time $t=0$. During the initialization phase, each sensor $u$ acquires the following local information: (i) the set $P_{u}$ of intersection points that it covers, (ii) the identities of the sensors in $T_{u}$ and (iii) the intersection points in $P_{u}$ that are covered by each sensor in $T_{u}$ (i.e., the set $P_{u, v}=P_{u} \cap P_{v}$ for each $v \in T_{u}$ ). As we elaborate in Section 5 , each sensor $u$ learns this information in a distributed manner by merely communicating with its neighbors and using only localized distance information. In addition, each sensor learns the following global network parameters: (i) $n$, the total number of sensors, and (ii) the maximum amount $B$ of the initial energy of any sensor

\footnotetext{
${ }^{2}$ Recall that the sensing range of each node is an open disc.
} 
( $B=\max _{u \in S} B_{u}$ ). Using the above information, each sensor computes $\mu$, where $\mu=4 n B$. The above constitutes the only global information each sensor needs to know throughout the execution of DLM, and can be communicated to each sensor using one network-wide broadcast.

\subsection{Activation phase}

The activation phase is executed at the beginning of each slot. We describe the computations in slot $j$.

Weight assignment: Let $b_{u}(j)$ be the energy of sensor $u$ that has been consumed in slots $1, \ldots j-1$. Then, at the beginning of slot $j$, sensor $u$ has already consumed $l_{u}(j)=$ $\frac{b_{u}(j)}{B_{u}}$ fraction of its energy. If $b_{u}(j)>B_{u}-1$, i.e., sensor $u$ does not have enough energy to monitor its sensing range throughout slot $j$, then it assigns itself a weight of $\infty$ at the beginning of slot $j$; otherwise it assigns itself a weight of $w_{u}(j)=\mu^{l_{u}(j)} / B_{u}$.

Sensor activation: Sensors that have infinite weights at the beginning of slot $j$ do not activate themselves in slot $j$. Among the rest, sensors are activated (using the DSC algorithm described in Section 4) so that the subset of activated sensors, $S(j)$, constitutes an $O(\log n)$-approximate minimum weight sensor cover. The sensors that do not activate themselves in slot $j$, sleep in slot $j$. Refer to Fig. 2 for a pseudo-code of the activation phase of DLM.

Intuitively, DLM has been designed so that the sensors are activated so as to cover the target field whenever possible, and the sensors that have large residual energy are preferentially selected. We will later prove that the lifetime of DLM is at least $\frac{1}{O((\log n)(\log n B))}$ times that of the maximum lifetime of the network.

When there does not exist any more, a sensor cover such that each sensor in the cover has non-zero energy, the network lifetime is considered terminated. After the network lifetime termination, we can not provide any guarantee on the target field coverage, although the sensors with finite weights continue to execute the algorithm, and cover their sensing ranges.

Note that each sensor can determine its weight based only on local information. In the next section, we show how each sensor can execute the activation phase using distributed computations based only on local information obtained from its neighbors.

The DLM Activation phase of sensor $u$ in slot $j$

begin
1: calculate $c_{u}(j)=\mu^{l_{u}(j)}$ and $w_{u}(j)=\frac{c_{u}(j)}{B_{u}}$ at the beginning of
slot $j$.
2: Use DSC in Fig. 3 to determine whether to stay active or enter
sleep mode.
end

Figure 2: The DLM Algorithm

\section{DISTRIBUTED SENSOR ACTIVATION}

We now describe an algorithm, which we call the Distributed Sensor Cover (DSC) algorithm, using which sensors can determine, using simple distributed computations, whether to activate themselves in each slot. Clearly, we need to design a sensor cover with guarantees on its weight using distributed computations. Note that a sensor cover is an instance of a set cover, and centralized algorithms that attain a $O(\log n)$-approximate set cover are well known [28]. We instead accomplish the same goal using distributed computations only, extending the design technique developed by Subhadrabandhu et al. [1] for the dominating set problem. We next describe our approach.

The sensor cover in each slot $j$ is iteratively computed in an asynchronous manner ${ }^{3}$. At the beginning of the activation phase in each slot, all the sensors with finite weights are contending for staying active in the slot. At any time during the activation phase, each contending sensor $u$, determines the number of intersection points in $P_{u}$ that have not yet been $k$-covered by the set of activated sensors, and computes its activation preference ratio $\left(a r_{u}\right)$ as the ratio between its weight in slot $j, w_{u}(j)$, and the above number. We denote by activation preference (ap) of sensor $u$, the ordered pair $a p_{u}=<a r_{u}, I D(u)>$, where $I D(u)$ is sensor $u$ 's ID. We say that sensor $u$ has lower ap than sensor $v$, i.e., $a p_{u}<a p_{v}$ if $a p_{u}$ has lower lexicographic value than $a p_{v}$, that is, (i) $a r_{u}<a r_{v}$ or (ii) $a r_{u}=a r_{v}$ and $I D(u)<I D(v)$. Each contending sensor $u$ communicates its activation preference to the sensors in $T_{u}$ at the beginning of the activation phase and each time that its value changes. Recall that the latter occurs only when one of $u$ 's neighbors in $T_{u}$ becomes active. A contending sensor $u$ activates itself once it detects that it has a lower activation preference than all contending sensors in $T_{u}$. Each sensor $u$ that activates itself informs other sensors in $T_{u}$, accordingly. Once a sensor $u$ detects that all the intersection points $P_{u}$ in its sensing range are $k$-covered by the already active sensors in $T_{u}$, it updates its neighbors and enters a sleep mode. The activation process, in each slot, terminates after each sensor decides whether to stay active or enter a sleep mode. Refer to Fig. 3 for a pseudo-code.

Clearly, each sensor can execute the above computations based only on locally available information, and the information it acquires in the Initialization phase (Subsection 3.1). Recall that a sensor $u$ enters a sleep mode only after all the intersection points $P_{u}$ in its sensing range are already $k$-covered. Thus, according to Theorem 1, during the lifetime of the network (i.e., while there is no coverage hole) the subset of sensors activated at the end of the activation phase in each slot $j, S(j)$, induces a sensor cover for the network. Moreover, we will later prove that $S(j)$ constitutes a $O(\log n)$-approximate minimum weighted sensor cover.

\section{THE INITIALIZATION PHASE}

During the initialization phase (Sub-Section 3.1), each sensor $u$ gains the knowledge of, (i) the set $P_{u}$ of intersection points that it covers, (ii) the identities of the sensors in $T_{u}$, which share intersection points with node $u$ and (iii) the set $P_{u, v}$ of the intersection points in $P_{u}$ that are covered by each sensor $v$ in $T_{u}$ (i.e., $P_{u, v}=P_{u} \cap P_{v}$ for each $v \in T_{u}$ ). We show that $u$ can determine the above using localized computations based on simple geometric properties. In these computations, $u$ only needs to know (a) $r_{u}$ (b) $N_{u}$, and their ids, (c) $d_{u, v}$ and $r_{v}$ for each $v \in N_{u}$ and (d) $d_{v, w}$ for each pair $v, w \in N_{u}$ such that $v$ and $w$ are neighbors of each other. We first provide a brief overview of the com-

\footnotetext{
${ }^{3}$ The sensors just need to know the beginning time of each time slot.
} 
The Distributed Sensor Cover (DSC) algorithm of sensor $u$

\section{Definitions:}

- Let $U C_{u} \subseteq P_{u}$ be the set of intersection points that have not yet been $k$-covered by the set of activated sensors.

- Let $C T_{u} \subseteq T_{u}$ be the set of contending neighbors of sensor $u$.

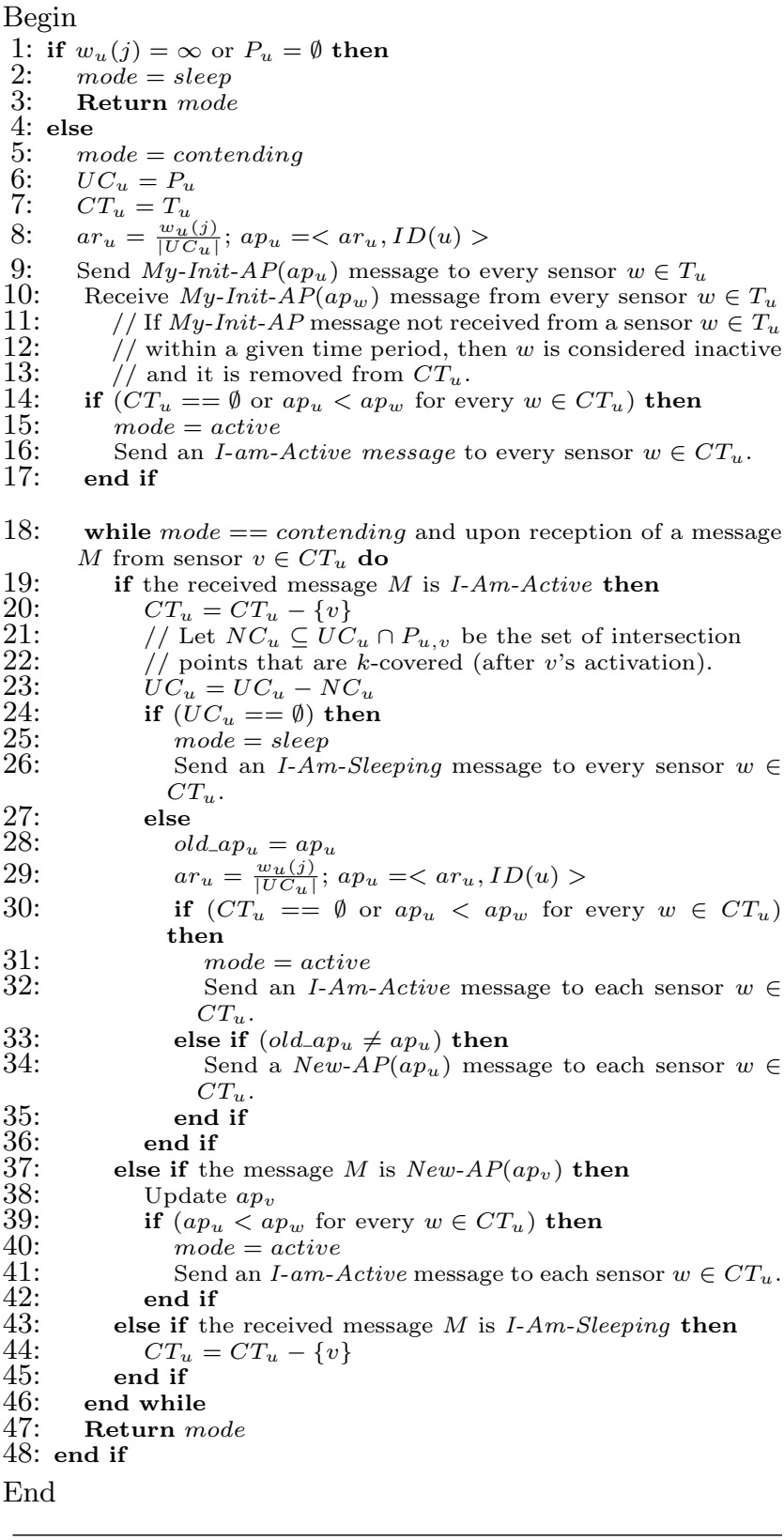

Figure 3: The Distributed Sensor Cover (DSC) algorithm.

putations in Sub-section 5.1 and subsequently present the details in Sub-section 5.2.

\subsection{Overview}

We assume that during the system activation every sensor $u$ initially evaluates its distance to each one of its neighbors in $N_{u}$ and it broadcasts these distances $d_{u, v}, v \in N_{u}$, as well as its sensing radius $r_{u}$ to its neighbors. Next, $u$ detects each neighbor $v \in N_{u}$ that intersects with $u$ by using Property 1 in Sub-section 2.4 and their joint intersection points. It also calculates the set $Q_{u}$ of all the intersection points of $u$ 's sensing border with the sensing borders of its neighbors. For every intersection point $p \in Q_{u}, u$ finds $S_{p}$, the set of sensors that cover $p$. Then, $u$ communicates these sets $S_{p}$, $p \in Q_{u}$ to its neighbors. This process enables every neighbor $v \in N_{u}$ of $u$ to know that a given intersection point $p \in Q_{u}$ is included in its sensing range and accordingly to add $p$ to its set $P_{v}$ of intersection points that it covers (for calculating (i) above). Moreover, the knowledge of each set $S_{p}, p \in P_{v}$, allows node $v$ to identify its neighbors $w$ that also cover each point $p \in P_{v}$ and update its set $T_{v}$ accordingly (for calculating (ii) above). Node $v$ can also calculate the sets $P_{v, w}=P_{v} \cap P_{w}$, for each $w \in T_{v}$ (for calculating (iii) above). Thus, to complete our description, we just need to present the process for detecting the set $Q_{u}$ of any given sensor $u \in S$ and calculating the set $S_{p}$ for every point $p \in Q_{u}$.

A major challenge in the initialization process is determining a unique identification for each intersection point. Since the sensors do not have any location information, the coordinates of the intersection points are unknown and cannot be used as identifiers. To overcome this difficulty, every intersection point of any pair $u, v$ of intersecting sensors is identified by a triplet $I P(u, v, i)$, where $u$ is the sensor with lower id, $v$ is the sensor with higher id, and $i=\{1,2\}$ denotes the point index. Since every pair $u, v$ of intersecting sensors have two common intersection points, the node with the lower id, say $u$, arbitrarily determines the index $i$ of each point. In addition, $u$ also calculates the set $S_{p_{i}}$ for both points $p_{i}=I P(u, v, i), i=\{1,2\}$ and communicates these sets to its neighbors, including node $v$. This ensures that each calculated set $S_{p}$ corresponds to a single intersection point that is uniquely defined. We describe the calculation of such sets $S_{p}$ in the next sub-section.

\subsection{Calculation of $S_{p}$}

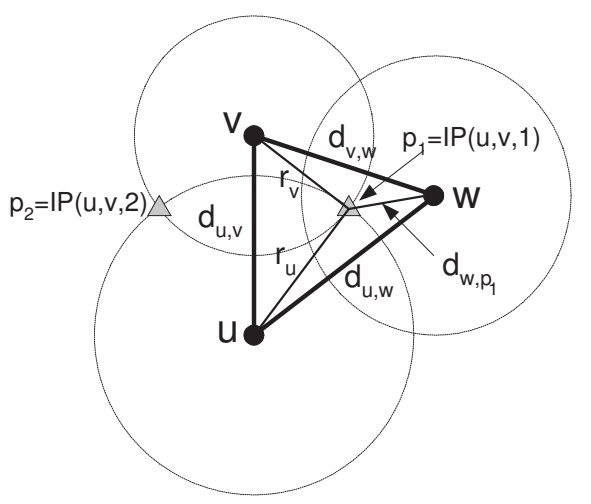

Figure 4: A pair of intersecting nodes and their intersection points.

Consider a pair $u, v$ of intersecting sensors and let $p_{i}=$ $I P(u, v, i), i=\{1,2\}$ denote their intersection points, as depicted in Figure 4 . We now describe a simple method for calculating the covering set $S_{p_{i}}, i=1,2$. First, note that a simple application of the triangle law on the distance metric 
and the fact that $\hat{R} \geq 2 \hat{r}$ establishes that $S_{p_{i}} \subseteq N_{u} \cap N_{v}$. Now, our calculation proceeds in two steps. In Step 1, we partition $N_{u} \cap N_{v}$ in three sets: nodes that cover (i) none, (ii) only one (iii) both of $p_{1}, p_{2}$. In Step 2, we identify which nodes among the second set in the above partition cover $p_{1}\left(p_{2}\right.$, respectively). This completes the computation of both $S_{p_{1}}, S_{p_{2}}$. In absence of location information, we rely on the Cosine Rule throughout. Let $d_{a, b}, d_{a, c}$ and $d_{b, c}$ denote the distances between three points $a, b$ and $c$ in a plane, accordingly, and let $\angle a, b, c$ denote the angle between the rays $[b, a]$ and $[b, c]$. The Cosine Rule states that

$$
2 \cdot d_{a, b} \cdot d_{b, c} \cdot \cos \angle a, b, c=d_{a, b}^{2}+d_{b, c}^{2}-d_{a, c}^{2}
$$

\subsubsection{Step 1}

For every node $w \in N_{u} \cap N_{v}$ that intersects with both $u$ and $v$, we check if it covers one or both of the points $p_{i}, i=\{1,2\}$. Recall that the straight line $(u, v)$ that traverses through the nodes $u, v$, partitions the plane into two halves, each one of which contains one of the points $p_{i}$, $i=\{1,2\}$. Without loss of generality, assume that $w$ is located in the same half as $p_{1}$ and therefore, it is closer to $p_{1}$ than to $p_{2}$. We first find the angle $\angle w, u, p_{1}$ as follows. As shown in Figure 4 , since $p_{1}$ and $w$ are in the same halfplane, $\angle w, u, p_{1}=\left|\angle v, u, w-\angle v, u, p_{1}\right|$ (recall that unlike the case presented in Figure $4, \angle w, u, p_{1}$ may also be equal to $\left.\angle v, u, p_{1}-\angle v, u, w\right)$. Since the distances $d_{u, v}, d_{u, w}, d_{v, w}$, $d_{u, p_{1}}=r_{u}$ and $d_{v, p_{1}}=r_{v}$ are known, the cosine rule applied on the triangles $\triangle u, w, v$ and $\triangle u, v, p_{1}$ enable us to calculate the angles $\angle v, u, w$ and $\angle v, u, p_{1}$, and hence the angle $\angle w, u, p_{1}$. Now, in $\triangle u, w, p_{1}$, the distance $d_{u, w}$ is known and $d_{u, p_{1}}=r_{u}$. So $d_{w, p_{1}}$ can be found using the Cosine rule. Thus, we can check whether $w$ covers $p_{1}$ by checking whether $d_{w, p_{1}}<r_{w}$.

Similarly, we check if $w$ covers $p_{2}$ by considering the triangle $\triangle u, w, p_{2}$. In this case the angle $\angle p_{2}, u, w=\angle v, u, w+$ $\angle v, u, p_{2}$. From symmetry, $\angle v, u, p_{2}=\angle v, u, p_{1}$. Thus, the angle $\angle p_{2}, u, w$ is known and $d_{w, p_{2}}$ can be calculated by the cosine rule in $\triangle u, w, p_{2}$. Note that this process can also be used to calculate the distances $d_{w, p_{1}}$ and $d_{w, p_{2}}$ if $w$ is located on the line $(u, v)$. In such case, $d_{w, p_{1}}=d_{w, p_{2}} ;$ thus $w$ covers both points or none of them.

\subsubsection{Step 2}

We now consider the set $Z \subseteq N_{u} \cap N_{v}$ of sensors that cover only one of the points $p_{1}, p_{2}$ and determine which sensors in $Z$ cover $p_{1}$. Consider an arbitrary sensor $w \in Z$ and, without loss of generality, let $p_{1}$ be the point that it covers. Now, for each sensor $x \in Z, x \neq w$, we check, as described next, whether $x$ covers $p_{1}$. As explained above, none of the nodes in $Z$ is located on the line $(u, v)$. Thus, all the sensors in $Z$ that are located in the same half plane as $w$ cover $p_{1}$, while the others cover $p_{2}$. Thus, we just need to check if $x$ and $w$ are in the same half. If $w$ and $x$ cover the same point, (and are therefore in the same half-plane), they must be neighbors. Thus, all the distances between every pair of nodes in $\{u, v, w, x\}$ are known and accordingly the three angles $\angle v, u, x, \angle v, u, w, \angle w, u, x$ can be calculated using the Cosine Rule. We use Property 3 to verify if $x$ and $w$ are in the same half-plane.

Property 3. Two sensors $w, x \in Z$ are located in the same half-plane, defined by the line $(u, v)$, if and only if (1) $\angle w, u, x=|\angle v, u, w-\angle v, u, x|$ and (2) $\angle w, u, x+\angle v, u, w+$ $\angle v, u, x<360^{\circ}$.

We omit the proof due to space constraints.

\section{DETECTION OF LIFETIME TERMINA- TION}

We now augment our scheme with a simple distributed mechanism for detecting the termination of the network lifetime. By definition the network lifetime terminates when there no longer exists a sensor cover such that every sensor in the cover has non-zero energy. Thus, from Theorem 1, the network lifetime ends once one of the intersection points in the IP set $P$ (Sub-Section 2.4) cannot be $k$-covered by the sensors that still have non-zero energy. Note that every point $p \in P$ is included in the closure of the sensing range of at least one internal node. Thus, once an internal node $u$ decides whether to remain active in the slot and knows the activity status of all its neighbors in $N_{u}$, it checks if each one of the intersection points in $P_{u} \cup Q_{u}$ is $k$-covered by the set of the active nodes in $N_{u} \cup\{u\}$. This is a simple test, as for every point $p \in\left(P_{u} \cup Q_{u}\right)$, the set $S_{p}$ of sensors that cover $p$ is already known ( Section 5). Node $u$ informs the administrators about the coverage hole once this test fails.

\section{SCHEME ANALYSIS}

We now prove correctness and performance guarantees for the DLM algorithm. In Subsection 7.1, we prove the guarantees for DSC which DLM invokes. Using the above, in Subsection 7.2 we prove the guarantees for DLM.

\subsection{DSC Algorithm- Analysis}

We prove that DSC computes an $O(\log n)$-approximate minimum weight sensor cover. Note that all the proofs allow for arbitrary, but finite transit times of status update messages transmitted by nodes to their neighbors.

Theorem 2. At every activation phase, (i) DSC computes a sensor cover if there is no coverage hole, (ii) DSC terminates in at most $2 n W$ time if $W$ is an upper bound on the transit delay of status update messages between the neighbors and (iii) DSC terminates in finite time, if the transit delays are finite but can not be upper-bounded.

Proof. First, note that at the beginning of the activation phase in a slot, each sensor $u$ knows its current ap and the current aps of the sensors in $T_{u}$. The contending sensor $v$ that has the minimum ap among all contending sensors knows that it has a lower ap than the sensors in $T_{v}$. Thus, $v$ activates itself at this time. Now, (ii) follows if we can show that while there exists at least one contending sensor, the difference between two consecutive activation times is at most $2 W$. Consider an arbitrary activation time $t$ of a sensor, say $x$. Let there be at least one contending sensor at $t+2 W$, and no sensor activate itself in $(t, t+2 W)$ (otherwise, there is nothing to prove). By time $t+W$, every sensor in $T_{x}$ learns that $x$ activated itself and transmits its updated ap to its neighbors. So, at $t+2 W$, every contending sensor $u$ knows its current ap and the current aps of the sensors in $T_{u}$. Thus, the contending sensor that has the minimum ap among all contending sensors activates itself at this time. Next, (iii) follows using a similar argument. Now, we prove (i). First consider the time $t$ in a given slot at which DSC has terminated. Let the target field not have a coverage hole. 
Then, each intersection point $w$ in $P$ is covered by $k$ or more contending sensors at the beginning of the activation phase. Since a contending sensor $u$ decides to sleep only when all the intersection points in $P_{u}$ are $k$-covered by activated sensors, all intersection points in $P$ are $k$-covered by activated sensors at $t$. The result follows by Theorem 1 .

Now, recall that finding a minimum weight sensor cover is an instance of the minimum weight set cover problem. We now describe the well-known greedy Centralized Set Cover (CSC) algorithm that computes a $O(\log n)$-approximate minimum weight set cover [28]. At each iteration, it selects the sensor which has the lowest activation preference (ap) among all the sensors, where ap is defined in the same way as for DSC, and then updates the ap's of the unselected sensors. This process continues until the set of selected sensors constitutes a sensor cover.

THEOREM 3. For a given setting and a set of weights to the sensors, DSC and CSC select the same set of sensors. Thus, DSC obtains an $O(\log n)$-approximate minimum weight sensor cover.

Proof. Let $Y^{C}=\left\{v_{1}, \cdots, v_{m^{C}}\right\}$ and $Y^{D}=\left\{u_{1}, \cdots, u_{m^{D}}\right\}$ be the sets of selected sensors by CSC and DSC, respectively, sorted in increasing order according to their ap values at the time that they were selected ${ }^{4}$ (i.e., decided to stay active). Let $v_{j}$ and $u_{j}$ be the $j$-th sensors in $Y^{C}$ and $Y^{D}$ respectively, and let $a p^{C}{ }_{j}$ and $a p^{D}{ }_{j}$ be their ap values. Moreover, let $Y^{C}{ }_{j}=\bigcup_{i=1, j} v_{j}$ and $Y^{D}{ }_{j}=\bigcup_{i=1, j} u_{j}$ be the first $j$ sensors in sets $Y^{C}$ and $Y^{D}$ respectively. Not that the sensors in $Y^{C}$ are arranged in the order in which they were selected by CSC. However, the order on the sensors in $Y^{D}$ is not necessarily the order in which they are activated by DSC.

Our proof utilizes the following properties:

(1) During the execution of DSC, the ap of each node is an increasing function of time.

(2) Consider any node $u \in Y^{D}$. Every sensor $w \in Y^{D} \cap T_{u}$ with lower ap value than $u$ was selected before $u$ by DSC. Similarly, any node $w \in Y^{D} \cap T_{u}$ with higher ap value than $u$ was selected after node $u$ by DSC.

This property follows from property (1) and from the fact that under DSC, a sensor $u$ becomes active only when (and if) it has lower ap value than its unselected neighbors in $T_{u}$. (3) The ap value of any node $u$ during the execution of CSC and DSC is determined only by its already selected neighbors in $T_{u}$.

(4) Suppose $u \in Y^{D}$ becomes active at time $t_{1}$ under DSC. Then, for each $w \in Y^{D} \cap T_{u}$ that became active before $t_{1}$, $u$ received an activation message from $w$ before time $t_{1}$.

If this were not true for some $w$, then note that $u$ would not have activated itself at $t_{1}$, since it would find its own ap to be higher than that of $w$.

We seek to prove that $Y^{C}=Y^{D}$. Let $Y^{C} \neq Y^{D}$ instead, and let $j$ be the lowest index such that $v_{j} \neq u_{j}$. Initially, let us show by contradiction that $j \leq \min \left(m^{C}, m^{D}\right)$. First, let $m^{C}>m^{D}$ and $j>m^{D}$. But, then, the first $m^{D}$ sensors selected by CSC constitute a sensor cover and therefore CSC terminates after selecting at most the first $m^{D}$ sensors. Now, let $m^{C}<m^{D}$ and $j>m^{C}$ (in particular $j=m^{C}+1$ ) and consider the vicinity of the node $u_{j}$. From Property (2),

\footnotetext{
$\overline{{ }^{4} \text { Here, by ap value of a node }} u \in Y^{D}$, we mean the latest
} ap value calculated by $u$. node $u_{j}$ was selected by DSC after every node in $Y^{D}{ }_{j-1} \cap$ $T_{u_{j}}=Y^{D}{ }_{m}{ }^{C} \cap T_{u_{j}}=Y^{C} \cap T_{u_{j}}$. However, since $Y^{C}$ is a sensor cover, all intersection points in $u_{j}$ 's sensing range are $k$-covered once DSC selects the nodes in $Y^{C} \cap T_{u_{j}}$. Thus, DSC does not select $u_{j}$ after it has selected the sensors in $Y^{D}{ }_{j-1} \cap T_{u_{j}}$, and thus it does not select $u_{j}$ at all. Thus, $j \leq \min \left(m^{C}, m^{D}\right)$.

We now show that $a p^{D}{ }_{j} \geq a p^{C}$. If not, let $a p^{D}{ }_{j}<a p^{C}{ }_{j}$ and consider the $j$-th iteration of CSC. The algorithm selects as the $j$-th active sensor, the unselected sensor with minimal ap value. Recall that at this stage $u_{j}$ has not been selected by CSC. Since $Y^{C}{ }_{j-1}=Y^{D}{ }_{j-1}$, from properties (2), (3) and (4) above, it follows that at the $j$-th iteration of CSC the ap value of node $u_{j}$ is the same as $a p^{D}{ }_{j}$ calculated by DSC. This is true since the ap value of node $u_{j}$ depends only on its selected neighbors in $Y^{C-1}{ }_{j-1} \cap T_{u_{j}}=Y^{D}{ }_{j-1} \cap T_{u_{j}}$, which are the same sets ${ }^{5}$ for both algorithms. Thus, CSC should select node $u_{j}$ rather than node $v_{j}$, which contradicts the assumption that $a p^{D}{ }_{j}<a p^{C}{ }_{j}$. Thus $a p^{D}{ }_{j} \geq a p^{C}{ }_{j}$.

We next show that $a p^{D}{ }_{j} \leq a p^{C}{ }_{j}$. If not, $a p^{D}{ }_{j}>a p^{C}{ }_{j}$. Since $v_{j}$ is in $Y^{C}$, it holds that nodes in $Y^{C}{ }_{j-1}$ do not cover all the intersection points covered by the node $v_{j}$. Thus, there are some nodes, denoted by set $W$, in the vicinity of $v_{j}$, i.e., $P_{w} \cap P_{v_{j}} \neq \emptyset \forall w \in W$, that were selected by DSC and are not in $Y^{D}{ }_{j-1}$. First, assume that $v_{j}$ is the first node in $W$ selected by DSC. From Property (3) above, $v_{j}$ 's ap value is determined only by the selected sensors in $Y^{D}{ }_{j-1}=Y^{C}{ }_{j-1}$. Thus, by Property (4), $v_{j}$ 's ap value at the time it is selected by DSC, is the same value as that at the time it is selected by CSC, i.e., $v_{j}$ 's ap value is $a p^{C}{ }_{j}$, which contradicts the assumption that $a p^{D}{ }_{j}>a p^{C}{ }_{j}$. Thus, $v_{j}$ is not the first node in $W$ selected by DSC. Let $x \in W, x \neq v_{j}$ be the first node in $W$ selected by DSC and let $a p^{D}{ }_{x}$ denote its ap value at the time it was selected by DSC, say time $t_{x}$. From our assumption, it follows that $a p^{D}{ }_{x} \geq a p^{D}{ }_{j}>a p^{C}{ }_{j}$. Now, consider the ap value of node $v_{j}$ as calculated by DSC just before time $t_{x}$ when node $x$ is selected. Since $x \neq v_{j}$ is the first node in $W$ selected by DSC, just before time $t_{x}$, the neighbors of $v_{j}$ selected by DSC must be from the set $Y^{D}{ }_{j-1}$ (all the neighbors of $v_{j}$ need not be in $\left.Y^{D}{ }_{j-1}\right)$. From Property (3), it holds that the ap value of $v_{j}$ is determined only by its selected neighbors. Thus, the ap value of $v_{j}$ just before time $t_{x}$ as calculated by DSC, denoted by $a p^{D}{ }_{v_{j}}$, is at most $a p^{C}{ }_{j}$. Thus, $a p^{D} v_{j} \leq a p_{j}{ }_{j}<a p^{D}{ }_{j} \leq a p^{D}{ }_{x}$. But, then, $v_{j}$ should have been selected by DSC rather than node $x$ and its ap value should have been $a p^{D}{ }_{v_{j}}$, which contradicts the assumption that $a p^{D}{ }_{j}>a p^{C}{ }_{j}$.

Thus, $a p^{D}{ }_{j}=a p^{C}{ }_{j}$. Hence, $I D\left(u_{j}\right)=I D\left(v_{j}\right)$. Thus, $u_{j}=v_{j}$, which is a contradiction. The result follows.

\subsection{DLM Algorithm- Analysis}

We now prove an approximation ratio for the lifetime attained by the Distributed Lifetime Maximization (DLM) algorithm in Fig. 2. Our analysis is similar to the ones used by Aspnes et al. [26] for online machine scheduling and virtual circuit routing problems, and Awerbuch et. al [4], [5] for the online virtual circuit routing problem.

Recall from Section 2.1 that a sensor that is active in a

\footnotetext{
${ }^{5}$ Note that by property (4), just before $u_{j}$ selected itself under DSC, it had updated its ap to account for the fact that all nodes in $Y^{D}{ }_{j-1} \cap T_{u_{j}}$ had activated themselves.
} 
slot consumes 1 unit of energy and a sensor in sleep mode consumes no energy. Throughout this section, all logarithms are to the base 2. Finally, for proving the approximation ratio, we additionally assume that the initial energy of each sensor is large enough:

Assumption 1. $B_{u} \geq \log \mu, u \in S$.

For simplicity, in the proof, we assume that $B_{u}, u \in S$ are integers. The proof can be easily extended to the case when they are real numbers.

\subsubsection{The DLM-T Algorithm}

We describe in Fig. 5 DLM-T (Truncated DLM), a modified version of DLM, that will be used to prove an approximation ratio for DLM.

\section{The DLM-T Algorithm}

\section{begin}

1: Let $c_{u}(j)=\mu^{l_{u}(j)}$ and $w_{u}(j)=\frac{c_{u}(j)}{B_{u}}$ be the weight of sensor $u$ at the beginning of slot $j$.

2: At the beginning of slot $j$ :

3: Using DSC in Fig. 3, find an $O(\log n)$-approximate minimum weight sensor cover $S(j)$ with weight:

$$
W(j)=\sum_{u \in S(j)} w_{u}(j)
$$

4: If $W(j) \leq 2 n$, then activate the sensor cover $S(j)$ in slot $j$, otherwise declare the network as dead. end

\section{Figure 5: The DLM-T Algorithm}

Note that DLM-T differs from DLM in the following: (i) the criterion it uses to declare the network as dead (step 4) (ii) it does not use a weight equal to $\infty$ for a sensor $u$ with 0 remaining energy, but a weight of $\frac{\mu}{B_{u}}$ (iii) it considers all nodes in the sensor cover selection process whereas DLM considers only those that have at least one unit of energy remaining. It is therefore not clear whether DLM-T selects nodes that have at least one unit of energy left. The next lemma however shows that this is indeed the case.

LEMMA 1. Under the DLM-T algorithm, if a sensor is activated at the beginning of slot $j$, it has at least one unit of energy remaining.

Proof. We need to show that for any $j \geq 1$, for any $u \in S(j), l_{u}(j) \leq 1-\frac{1}{B_{u}}$. Note that $W(j) \leq 2 n$. Thus, for any such $u$,

$$
w_{u}(j) \leq W(j) \leq 2 n
$$

Hence,

$$
\mu^{l_{u}(j)}=B_{u} w_{u}(j) \leq 2 n B_{u} \leq 2 n B=\frac{\mu}{2}=\mu^{1-\frac{1}{\log \mu}}
$$

where the last equality follows since the logarithms are to the base 2 . So,

$$
l_{u}(j) \leq 1-\frac{1}{\log \mu} \leq 1-\frac{1}{B_{u}}
$$

by Assumption 1. The result follows.

The next result establishes the relation between the lifetimes of the DLM and DLM-T algorithms.
LEMma 2. The lifetime of the network under the DLM algorithm is greater than or equal to that under DLM-T.

Proof. Consider two identical networks- one running DLM and the other running DLM-T. We show that both networks run identically until the beginning of slot $j^{\prime}$, which is the first slot when the sensor cover $S\left(j^{\prime}\right)$ found by DLM-T has weight $W\left(j^{\prime}\right)>2 n$. We show this by induction. In the first slot, every sensor has at least 1 unit of energy. Since DLM and DLM-T use the same weights and the DSC algorithm, both algorithms select the same sensor cover. As induction hypothesis, suppose both algorithms select the same sensor cover in slots $1, \ldots, j-1$, where $j \leq j^{\prime}-1$.

Now, we show that the sensor cover $\tilde{S}(j)$ found by DLM in slot $j$ is the same as the sensor cover $S(j)$ found by DLM-T. At the beginning of slot $j$, let $X \subseteq S$ be the set of sensors with at least 1 unit of energy, and $Y=S \backslash X$ be the set of sensors in $S$ with 0 energy. Now, since $j \leq j^{\prime}-1$, in slot $j$, in the sensor cover $S(j)$ selected by DLM-T, no sensor has 0 energy by Lemma 1 . Hence:

$$
S(j) \subseteq X
$$

Note that DLM runs DSC on the set of sensors $X$ and DLM-T runs DSC on the set of sensors $X \cup Y=S$. By Theorem 3, the DSC algorithm run by DLM (respectively, DLM-T) finds the same set of sensors as the Centralized Set Cover (CSC) algorithm running on the set of sensors $X$ (respectively, $X \cup Y$ ). It is therefore sufficient to show that if DLM and DLM-T were to use CSC (instead of DSC) in slot $j$, they would have selected the same sensor cover in slot $j$. Denote the CSC algorithm corresponding to the network running DLM-T by CSC-T to distinguish it from the CSC algorithm corresponding to the network running DLM (denoted simply by CSC).

Now, we show by induction on the iterations of CSC and CSC-T, that each algorithm selects the same set $S(j)$. Let $u_{i}$ (respectively, $w_{i}$ ) be the sensor selected by CSC-T (respectively, CSC), in the $i$ 'th iteration. At the beginning of the first iteration, the ap of each node $u \in X$ is the same in CSC and CSC-T (it equals $<\frac{w_{u}(j)}{P_{u}}, I D(u)>$ ). Since $u_{1} \in S(j) \subseteq X$ by (2), $u_{1}$ is the node with lowest ap in $X \cup Y$ and hence in $X$. So CSC selects $u_{1}$, i.e., $w_{1}=u_{1}$. As induction hypothesis, assume that $w_{2}=u_{2}, \ldots, w_{i-1}=u_{i-1}$.

Now, consider the beginning of the $i$ 'th iteration in CSC-T and CSC. Recall that the ap value of a node under the CSC and CSC-T algorithms depends only on the set of already selected sensors [28], which is the same in both algorithms. Also, by (2), $u_{i} \in X$ and it is the node with lowest ap among the unselected nodes in $X \cup Y$ and hence in $X$. It follows that CSC selects $u_{i}$ in iteration $i$, i.e., $w_{i}=u_{i}$. This completes the induction on $i$ and hence $\tilde{S}(j)=S(j)$.

This, in turn, completes the induction on $j$ and hence we have shown that the networks running DLM and DLM-T run identically until the beginning of slot $j^{\prime}$. At the beginning of slot $j^{\prime}$, the network running DLM-T declares itself dead, while the network running DLM possibly continues. This concludes the proof.

Note that unlike DLM, DLM-T requires not only the determination of a $O(\log n)$-approximate minimum weight sensor cover, but also the calculation of its weight. The latter requires network-wide coordination. Nevertheless, it follows from Lemma 2 that any approximation ratio that holds for the lifetime of DLM-T, holds for DLM as well. We therefore 
prove an approximation ratio for DLM, by proving one for DLM-T next.

\subsubsection{Approximation Ratio}

Let $O P T$ be an optimal algorithm for the maximum lifetime problem, $L$ be the network lifetime under the DLM-T algorithm and $L^{*}$ be the network lifetime under OPT. Also, let $\mathcal{L}=\{1, \ldots, L\}$ be the set of slots when the network is alive under the DLM-T algorithm and $\mathcal{L}^{*}=\left\{L+1, \ldots, L^{*}\right\}$ be the set of slots when the network is dead under the DLM$\mathrm{T}$ algorithm, but alive under OPT.

We can view the situation after the network dies under DLM-T as if at the beginning of every slot $j \in \mathcal{L}^{*}$, the network finds an approximate minimum weight sensor cover (it finds the same sensor cover for each $j \in \mathcal{L}^{*}$ ) and since the weight of this cover is greater than $2 n$, it does not activate it. Under DLM-T, no sensor is activated after slot $L$ and hence the weights of all sensors remain unchanged thereafter.

Let $S(j)$ be the sensor cover found by DLM-T and $S^{*}(j)$ be the sensor cover used by OPT in slot $j$. Also, let $W(j)$ be the weight of $S(j)$ and $W^{*}(j)$ be the sum of the weights of the sensors in $S^{*}(j)$ at the beginning of slot $j$ when the network is running $D L M-T$. We emphasize that the sensor cover $S^{*}(j)$ is the one used by OPT in slot $j$, but the weights of the sensors in $W^{*}(j)$ are those when the network is running DLM-T.

Now, in every slot, the DLM-T algorithm finds an $O(\log n)$ approximate minimum weight sensor cover. Hence, there exists a constant $\alpha$ such that:

$$
W(j) \leq(\alpha \log n) W^{*}(j)
$$

The following theorem proves the approximation ratio achieved by the DLM-T algorithm.

THEOREM 4. $L^{*}$ is at most an $O((\log n)(\log \mu))$ factor greater than $L$.

The proof proceeds as follows. We first upper bound the amount by which the network lifetime under OPT can exceed that under the DLM-T algorithm (Lemma 3). Next, we lower bound the lifetime achieved by DLM-T (Lemma 4). Finally, we obtain an upper bound on the ratio $\frac{L^{*}}{L}$ by combining the above bounds.

\section{LEMMA 3.}

$$
L^{*}-L \leq \frac{\alpha \log n}{2 n} \sum_{u \in S} c_{u}(L+1)
$$

Proof. We define the indicator function:

$$
I\left\{u \in S^{*}(j)\right\}=\left\{\begin{array}{rr}
1 & \text { if } u \in S^{*}(j) \\
0 & \text { else }
\end{array}\right.
$$

Since $W(j)>2 n$ for $j \in \mathcal{L}^{*}$, from (3) it follows that:

$$
W^{*}(j) \geq \frac{2 n}{\alpha \log n} \forall j \in \mathcal{L}^{*}
$$

Summing the above over $j \in \mathcal{L}^{*}$, we get:

$$
\sum_{j \in \mathcal{L}^{*}} W^{*}(j) \geq \frac{2 n}{\alpha \log n}\left(L^{*}-L\right)
$$

Hence,

$$
\begin{aligned}
& \frac{2 n}{\alpha \log n}\left(L^{*}-L\right) \\
\leq & \sum_{j \in \mathcal{L}^{*}} \sum_{u \in S^{*}(j)} \frac{1}{B_{u}} c_{u}(j) \\
= & \sum_{j \in \mathcal{L}^{*}} \sum_{u \in S^{*}(j)} \frac{1}{B_{u}} c_{u}(L+1) \\
= & \sum_{j \in \mathcal{L}^{*}} \sum_{u \in S} \frac{c_{u}(L+1)}{B_{u}} I\left\{u \in S^{*}(j)\right\} \\
= & \sum_{u \in S} c_{u}(L+1)\left[\frac{1}{B_{u}} \sum_{j \in \mathcal{L}^{*}} I\left\{u \in S^{*}(j)\right\}\right] \\
= & \sum_{u \in S} c_{u}(L+1)
\end{aligned}
$$

where in (5), we used the fact that since the network is dead under DLM-T at the beginning of slot $L+1$, the energy of each sensor remains same thereafter and hence $c_{u}(j)=c_{u}(L+1) \forall j \in \mathcal{L}^{*}$. We get (6) from the fact that $\sum_{j \in \mathcal{L}^{*}} I\left\{u \in S^{*}(j)\right\}$ is the number of slots in $\mathcal{L}^{*}$ in which sensor $u$ is activated under OPT and must not exceed the initial energy $B_{u}$ of the sensor.

LEMMA 4.

$$
\sum_{u \in S} c_{u}(L+1) \leq n(2 L \log \mu+1)
$$

Proof. We begin by upper bounding the total growth in the functions $c_{u}($.$) of sensors u \in S(j)$ over slot $j$. For slot $j \in \mathcal{L}$, we have:

$$
\begin{aligned}
\sum_{u \in S(j)}\left(c_{u}(j+1)-c_{u}(j)\right) & =\sum_{u \in S(j)}\left(\mu^{l_{u}(j)+\frac{1}{B_{u}}}-\mu^{l_{u}(j)}\right) \\
& =\sum_{u \in S(j)} \mu^{l_{u}(j)}\left(2^{\frac{\log \mu}{B_{u}}}-1\right) \\
& \leq \sum_{u \in S(j)} \mu^{l_{u}(j)}\left(\frac{\log \mu}{B_{u}}\right) \\
& =\log \mu \sum_{u \in S(j)} \frac{\mu^{l_{u}(j)}}{B_{u}} \\
& \leq 2 n \log \mu
\end{aligned}
$$

where (8) results from the facts that $\frac{\log \mu}{B_{u}} \leq 1$ by Assumption 1 and $2^{x}-1 \leq x \forall x \in[0,1]$. Inequality (9) follows from:

$$
\sum_{u \in S(j)} \frac{\mu^{l_{u}(j)}}{B_{u}} \leq 2 n
$$

which is true because the network is not declared dead by DLM-T at the beginning of slot $j$.

Now, in slot $j$, the energy of sensors $u \notin S(j)$ does not change and hence $c_{u}(j+1)=c_{u}(j) \forall u \notin S(j)$. So we get:

$$
\begin{aligned}
\sum_{u \in S}\left(c_{u}(j+1)-c_{u}(j)\right) & =\sum_{u \in S(j)}\left(c_{u}(j+1)-c_{u}(j)\right) \\
& \leq 2 n \log \mu
\end{aligned}
$$

Summing this inequality over $j \in \mathcal{L}$ :

$$
\sum_{j=1}^{L} \sum_{u \in S}\left(c_{u}(j+1)-c_{u}(j)\right) \leq 2 n L \log \mu
$$


The left hand side is a telescoping sum. So we get:

$$
\sum_{u \in S} c_{u}(L+1) \leq 2 n L \log \mu+\sum_{u \in S} c_{u}(1)
$$

But $c_{u}(1)=\mu^{0}=1 \forall u \in S$. Thus,

$$
\sum_{u \in S} c_{u}(L+1) \leq n(2 L \log \mu+1)
$$

Proof of Theorem 4. By Lemmas 3 and 4:

$$
L^{*} \leq L(\alpha(\log n)(\log \mu)+1)+\frac{\alpha \log n}{2}
$$

The result follows since $\alpha$ is a constant.

\section{SIMULATIONS}

We now evaluate the performance of DLM using simulations. We consider a WSN with $n$ sensors, each with an initial energy of $B$ units, sensing and transmission radii of 10 and 22 units respectively, deployed uniformly at random in a $50 \times 50$ units $^{2}$ target field, and examine the lifetime attained by DLM as functions of $n$ and $B$. Each time slot was 1 unit long.

We compared the lifetimes of the network under three algorithms: the DLM algorithm (Fig. 2), the Garg-Konemann (GK) algorithm [11] and a heuristic proposed in [13, 21] that we denote by Min-Num. At every slot, Min-Num finds a sensor cover with the minimum number of nodes (up to an $O(\log n)$ factor) and activates it. GK [11] generates a sequence of sets of weights to assign to the sensors and finds minimum weight sensor covers for each set of weights. When the initial energy of each sensor is the same, each sensor cover selected by GK is activated for an equal amount of time, which is a monotonically increasing function of an input parameter $\epsilon$. Thus, the number of sensor cover computations per slot, and hence the computation time required for GK, increases as $\epsilon$ decreases. The lifetime approximation ratio guaranteed for the GK algorithm however worsens with increase in $\epsilon^{6}$.

First, we plot in Fig. 6, lifetimes achieved by DLM, MinNum, GK as a function of $n$, for $B=15$. For GK, we select (i) $\epsilon$ such that it computes sensor covers at the same rate per unit time as DLM, Min-Num (i.e., approximately once every slot) (denoted by GK(1 slot)) and (ii) $\epsilon=0.1$ (denoted by $\operatorname{GK}(\epsilon=0.1))$. In (ii), GK computes sensor covers at least 32 times per slot on an average for the range of $n$ we considered. Next, in Fig. 7, we plot the lifetimes of DLM, MinNum and GK(1 slot) ${ }^{7}$ as a function of $B$ for $n=150$ and $\epsilon$ such that GK computes a sensor cover approximately once every slot. The figures reveal that GK and DLM perform similarly only when GK computes sensor covers much more frequently than DLM, and DLM outperforms GK otherwise. Thus, although GK guarantees a better approximation ratio (while using centralized computation and location information), in practice, DLM outperforms GK. DLM substantially

\footnotetext{
${ }^{6}$ The network lifetime under the GK algorithm is guaranteed to be at most a factor $(1+\epsilon) f$ less than the optimal lifetime, where $f=O(\log n)$ is the approximation ratio of the algorithm used for finding minimum weight sensor covers [11].

${ }^{7}$ The values of $\epsilon$ used for $\mathrm{GK}(1$ slot $)$ lie in $[0.67,0.71]$ for Fig. 6 and in $[0.35,0.70]$ for Fig. 7 .
}

out-performs Min-Num as well, which suggests that lifetime can be substantially enhanced by deciding which sensors to activate based on their residual energy.

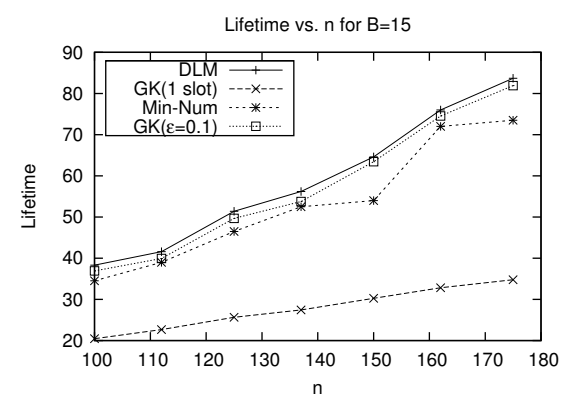

Figure 6: Plot of lifetimes of DLM, GK (1 slot), Min-Num and GK $(\epsilon=0.1)$ vs. $n$ for $B=15$ units

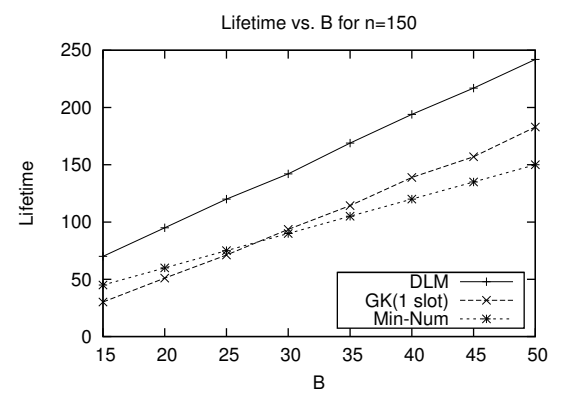

Figure 7: Plot of lifetimes of DLM, GK(1 slot) and Min-Num vs. $B$ for $n=150$

Recall that DLM assigns weights to sensors using a parameter $\mu$. In order to study the sensitivity of DLM to the value of $\mu$, we fixed the values of $n$ and $B$ and plot in Fig. 8, the lifetime of DLM using values of $\mu$ between 2 and 32000 . The plot shows that the lifetime achieved by DLM is roughly

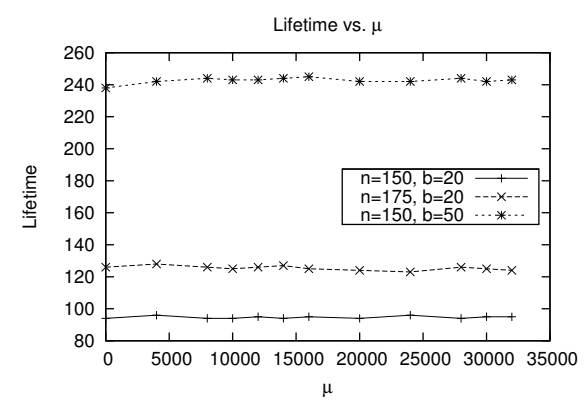

Figure 8: Plot of lifetimes of the DLM algorithm vs. $\mu$ for $(n=150, B=20),(n=175, B=20)$ and $(n=150, B=50)$

the same for different values of $\mu$. This suggests that high lifetimes can be attained even without selecting $\mu=4 n B$, as required for proving the approximation guarantee. Thus, in practice, the sensors need not exchange any global information (specifically, $n, B$ ) even during the initialization phase. 


\section{CONCLUSION}

We designed a distributed, coordinate-free algorithm for attaining high lifetimes in sensor networks, subject to ensuring the $k$-coverage of the target field during the network lifetime. We proved that the lifetime attained by our algorithm approximates the maximum possible lifetime within a logarithmic approximation factor. Simulation results reveal that our algorithm substantially outperforms other schemes for lifetime maximization.

\section{ACKNOWLEDGMENTS}

The contributions of G. Kasbekar and S. Sarkar have been supported by NSF grants NCR-0238340, CNS-0721308, ECS0622176 .

\section{REFERENCES}

[1] D. Subhadrabandhu, F. Anjum, S. Kannan, S. Sarkar "Domination and Coverage Guarantees Through Distributed Computation" in Proceedings of $43 d$ Annual Allerton Conference on Communication, Control and Computing, Allerton, Monticello, Illinois, September 28-30, 2005

[2] C-F. Huang and Y.-C Tseng, "The Coverage Problem in a Wireless Sensor Network", In Proc. of $A C M$ WSNA'03, Sep. 2003.

[3] G. Kasbekar, Y. Bejerano and S. Sarkar, "Generic Coverage Verification without Location Information Using Dimension Reduction" in Proc. of Wiopt'09, Seoul, June 2009

[4] B. Awerbuch, Y. Azar, S. Plotkin "Throughput-Competitive On-Line Routing" in Proceedings of IEEE Symposium on Foundations of Computer Science, 1993.

[5] S. Leonardi "On-Line Network Routing" in Online Algorithms- the State of the Art, ed. A. Fiat and G. Woeginger, 1998.

[6] C. Zhang, Y. Zhang and Y. Fang "Detecting Coverage Boundary Nodes in Wireless Sensor Networks", In Proc. of ICNSC '06, April 2006.

[7] Y. Wang, J. Gao, J. S. B. Mitchell, "Boundary Recognition in Sensor Networks by Topological Methods", In Proc. of Mobicom '06, Sep. 2006.

[8] B. Alavi and K. Pahlavan "Modeling of the TOA-based Distance Measurement Error Using UWB Indoor Radio Measurements", In IEEE Communications Letters, Vol. 10, No. 4, April 2006, pp. 275-277.

[9] C. Y. Wen, R. D. Morris, and W. A. Sethares, "Distance Estimation Using Bidirectional Communications Without Synchronous Clocking", Accepted for publication in IEEE Trans. Signal Processing.

[10] Y. Bejerano, "Simple and Efficient $k$-Coverage Verification without Location Information". In Proc. of Infocom'08, Phoenix, Arizona, U.S.A., April 2008.

[11] P. Berman, G. Calinescu, C. Shah and A. Zelikovsly. "Efficient energy management in sensor networks". In Y. Xiao \& Y. Pan (Eds.), Ad hoc and sensor networks. Nova Science, 2005.

[12] F. Zhao and L. Guibas, "Wireless Sensor Networks: An Information Processing Approach". Morgan Kaufmann, 2004.
[13] M. Cardei, M.T. Thai, Y. Li, W. Wu "Energy-Efficient Target Coverage in Wireless Sensor Networks". In Proc. of Infocom'05, Miami, U.S.A., March 2005.

[14] M. Cardei and J. Wu. "Coverage in Wireless Sensor Networks", Handbook of Sensor Networks. CRC Press 2004.

[15] L. Wang and Y. Xiao "A Survey of Energy-Efficient Scheduling Mechanisms in Sensor Networks" In Mobile Networks and Applications, Vol. 11, pp. 723-740, 2006.

[16] R. R. Choudhury and R. Kravets, "Location-Independent Coverage in Wireless Sensor Networks", Technical Report, UIUC, 2004

[17] R. Ghrist, A. Muhammad, "Coverage and hole-detection in sensor networks via homology". In Proc. of IPSN 2005, April 2005.

[18] A. Man-Cho So and Y. Ye. "On Solving Coverage Problems in a Wireless Sensor Network Using Voronoi Diagrams". In Proc. of WINE 2005, LNCS 3828, pp. 584-593, 2005.

[19] S. Meguerdichian, F. Koushanfar, M. Potkonjak, M. B. Srivastava, "Coverage Problems in Wireless Ad-hoc Sensor Networks". In Proc. of Infocom'01, Anchorage, Alaska, U.S.A., April 2001.

[20] H. Zhang and J. C. Hou, "Maintaining sensing coverage and connectivity in large sensor networks". In International Journal of Wireless Ad Hoc and Sensor Networks, vol. 1, num. 1-2, pp. 89-123, January 2005.

[21] X. Wang, G. Xing, Y. Zhang, C. Lu, R. Pless, and C. Gill. "Integrated coverage and connectivity configuration in wireless sensor networks". In Proc. of ACM SenSys'03, Los Angeles, CA, Nov. 2003.

[22] Y. Wu, S. Fahmy, N. Shroff, "On the Construction of a Maximum-Lifetime Data Gathering Tree in Sensor Networks: NP-Completeness and Approximation Algorithm" In Proc. of Infocom'08, Phoenix, Arizona, U.S.A., April 2008.

[23] D. Niculescu, "Positioning in ad hoc sensor networks", In IEEE Network, Volume 18, Issue 4, July-Aug. 2004, pp. 24-29

[24] N. Patwari, J. N. Ash, S. Kyperountas, A. O. Hero, R.L. Moses and N. S. Correal, "Locating the nodes: cooperative localization in wireless sensor networks", In IEEE Signal Processing Magazine, Volume 22, Issue 4, July 2005 pp. 54-69

[25] Q. Shi, S. Kyperountas, N. S. Correal and F. Niu. "Performance Analysis of Relative Location Estimation for Multihop Wireless Sensor Networks". IEEE Journal On Selected Areas In Communications (JSAC), Vol 23, No. 4, April 2005.

[26] J. Aspnes, Y. Azar, A. Fiat, S. Plotkin, and O. Waarts. "On-line load balancing with applications to machine scheduling and virtual circuit routing". In Proc. ACM STOC, pp. 623-631, 1993.

[27] W. Rudin, "Principles of Mathematical Analysis", Mc-Graw Hill, Third Edition, 1976.

[28] V. Chvatal, "A Greedy Heuristic for the Set-Covering Problem", Mathematics of Operations Research, Vol. 4, No. 3 (Aug., 1979), pp. 233-235. 